\title{
Resin Infiltration of Artificial Enamel Caries Lesions with Experimental Light Curing Resins
}

\author{
Sebastian PARIS ${ }^{1}$, Hendrik MEYER-LUECKEL ${ }^{1}$, Helmut CÖLFEN ${ }^{2}$ and Andrej M. KIELBASSA ${ }^{1}$ \\ ${ }^{1}$ Department of Operative Dentistry and Periodontology, University School of Dental Medicine, Campus Benjamin Franklin, \\ Charité - Universitätsmedizin Berlin, Assmannshauser Str. 4-6, 14197 Berlin, Germany \\ ${ }^{2}$ Department of Colloid Chemistry, Max Planck Institute of Colloids and Interfaces, Am Mühlenberg 1, 14476 Potsdam-Golm, \\ Germany \\ Corresponding author, Hendrik MEYER-LUECKEL; E-mail: hendrik.meyer-lueckel@charite.de
}

Received December 19, 2006/Accepted April 2, 2007

To prevent enamel lesions from further demineralization, a complete and homogeneous penetration of low-viscosity resins ('infiltrants') should be accomplished. With commercially available adhesives, this goal might not be achieved because of their penetration capabilities. On this note, the Penetration Coefficient (PC) describes the penetrativity of liquids and might be employed to develop optimal infiltrants. Thus, the aim of this study was to compare the penetration abilities of 12 experimental infiltrants (BisGMA/TEGDMA comonomers showing varying PCs) with a commercially available adhesive (Excite, Vivadent). In each of the 156 bovine enamel specimens, four subsurface lesions were created. Three of the four lesions were infiltrated with either the adhesive or one of 12 experimental resins for either 10, 22, or 40 seconds, and subsequently light-cured. Specimens were studied using confocal microscopy and penetration depths were determined. A good correlation between PC and penetration depth was thereby observed (Pearson's correlation coefficient, $\mathrm{r}=0.820$ ).

Keywords: Caries, Infiltration, Infiltrant

\section{INTRODUCTION}

Despite a general decline of dental decay in the western industrialized nations in the past few decades, the prevalence of enamel caries is still comparably high ${ }^{11}$. The common treatment strategy for enamel caries lesions either focuses on remineralization by removing dental plaque and topical application of fluoride, or by invasive treatment regimes. However, remineralizing therapy fails when plaque is not sufficiently removed (e.g., due to cavitation in the proximal space or lack of compliance $)^{2)}$. In situations as such, operative treatment is indicated if caries progression cannot be hampered. In particular for proximal surfaces, large areas of sound enamel have to be removed even with minimally invasive preparation techniques.

An alternative therapy to arrest initial caries lesions would be the infiltration of the latter using light curing resins ${ }^{3,4}$. The pores within enamel lesions provide diffusion pathways for acids and dissolved minerals. The aim of caries infiltration is to occlude these pores, and thus prevent acid penetration into the lesions. In contrast to fissure sealing (where the diffusion barrier is established on the enamel surface), the present technique aims to create the diffusion barrier inside the enamel lesions. Moreover, the resin matrix could strengthen the enamel structure, thereby preventing cavitation or breakdown of the enamel surface. For initial enamel lesions on smooth surfaces, the application of infiltrating resins might postpone the first operative intervention for some years. Therefore, there exists an optimistic possibility that resin infiltration into enamel lesions might be clinically applicable in operative dentistry.

To date, several in vitro studies have investigated the penetration of light curing resins into artificial enamel lesions ${ }^{3,5-8)}$. It was demonstrated that infiltrated lesions progressed significantly slower in a demineralizing environment as compared to untreated lesions. Moreover, completely infiltrated lesions showed less progression compared to only superficially infiltrated lesions $\mathrm{s}^{4,9}$.

In most studies concerning resin infiltration, commercially available dental adhesives and fissure sealants were used to infiltrate enamel caries $^{3,5-8)}$. Although a complete infiltration of shallow lesions could be accomplished using these products, penetration of natural, deeper lesions could be achieved at most superficially ${ }^{10}$. Since adhesives and fissure sealants have been developed mainly with regard to adhesion ${ }^{11,12)}$, it is not surprising that these materials show inferior penetration capabilities.

Therefore, light curing resins - optimized for rapid penetration into enamel caries lesions (socalled 'infiltrants') - might be a preferred option ${ }^{13}$. In an early study, a low-viscosity resin, resorcinolformaldehyde, has been shown to be able to infiltrate caries lesions effectively ${ }^{14)}$. However, this material is not clinically applicable due to its insanitary nature. Therefore, fast-penetrating light curing resins remain to be explored and developed.

A physical model that describes the penetration 
of a liquid (light curing resin) into a porous solid (enamel lesion) is the Washburn equation (Equation $1)^{15)}$. This simplified model describes the porous solid as a bundle of open capillaries. The liquid penetrates into the solid driven by capillary forces. The bracketed term of the Washburn equation is the Penetration Coefficient (PC; Equation 2) $)^{16,17)}$. It describes the liquid characteristics of surface tension, viscosity, and contact angle to the solid phase. The higher the $\mathrm{PC}$ of a liquid, the faster it penetrates into a given porous solid. If the Washburn equation is applicable for the infiltration of enamel lesions, then optimal infiltrants could be developed by leveraging on the $\mathrm{PC}$ values of experimental resin mixtures.

$$
\begin{aligned}
& d^{2}=\left(\frac{\gamma \cdot \cos \theta}{2 \eta}\right) r \cdot t \\
& P C=\left(\frac{\gamma \cdot \cos \theta}{2 \eta}\right)
\end{aligned}
$$$$
\text { Equation } 1
$$

$d$ distance moved by the liquid resin

$\theta$ contact angle of liquid resin (to enamel)

$\mathrm{Y}$ surface tension of liquid resin (to air)

$\eta$ dynamic viscosity of liquid resin

$t$ penetration time

$r$ capillary (pore) radius

In a previous investigation, a positive correlation between the PCs of fissure sealants and the penetration into fissures was found ${ }^{17)}$. Furthermore, a recent study was able to determine the PCs of commercially available adhesives and experimental resin mixtures intended to infiltrate enamel lesion $\mathrm{s}^{13)}$. Commercially available adhesives revealed relatively low PCs, whereas some experimental resins yielded PCs tenfold higher. However, in terms of penetration speed into the enamel lesions, it remains to be known if the $\mathrm{PC}$ of a light curing resin can be used to predict this parameter.

The aim of the present investigation was to evaluate in vitro the penetration abilities of 12 experimental infiltrants and one commercially available adhesive into artificial enamel caries lesions. By employing the Washburn equation to assess the infiltration of resins into initial artificial enamel caries lesions, this study also served to prove the applicability of this model. The working hypothesis was that penetration depth (PD) correlates linearly with the square root of the product of the $\mathrm{PC}$ of an infiltrant and penetration time (i.e., $P D \sim \sqrt{P C \cdot t}$ ).

\section{MATERIALS AND METHODS}

Enamel specimens

From the facial aspect of bovine incisors, enamel specimens (approximately $6 \times 4 \times 4 \mathrm{~mm}^{3}$ ) were prepared
(Band Saw Exakt 300cl, Exakt Apparatebau, Norderstedt, Germany) and embedded in acrylic resin (Technovit 4071, Heraeus Kulzer, Hanau, Germany). Enamel surfaces were ground flat and polished with up to 4000-grit abrasive paper (Polishing Machine Phoenix Alpha, Buehler, Düsseldorf, Germany; Abrasive Paper, Exakt Apparatebau). Subsequently, acid-resistant nail varnish was applied on parts of the enamel surface (sound control), leaving four windows unprotected (A, B, C, D).

To create artificial enamel lesions in the uncovered areas, specimens were exposed to five liters of a demineralizing solution containing $50 \mathrm{mM}$ acetic acid, $3 \mathrm{mM} \mathrm{CaCl} \mathrm{m}_{2} \mathrm{H}_{2} \mathrm{O}, 3 \mathrm{mM} \mathrm{KH}_{2} \mathrm{PO}_{4}, \quad 6 \mu \mathrm{M}$ methylhydroxydiphosphonate ${ }^{18)}$ for 50 days ( $\mathrm{pH} 5.0$; $37^{\circ} \mathrm{C}$ ). The $\mathrm{pH}$ was checked daily, and if necessary, adjusted with a small amount of either hydrochloric acid $(10 \%)$ or potassium hydroxide solution (10 M) (all chemicals from Sigma-Aldrich, Steinheim, Germany).

After the abovementioned period of 50 days, each specimen showed four enamel lesions. To prepare the enamel surface for infiltration, three lesions (B, C, D) were etched with $37 \%$ phosphoric acid gel (Total Etch, Ivoclar Vivadent, Schaan, Liechtenstein) for five seconds, whereas one lesion served as untreated control (A). After rinsing the etching gel for 30 seconds (dental triple syringe), specimens were dried by immersion in ethanol (100\%) for 10 seconds and subsequent air-blowing for 30 seconds (dental triple syringe).

\section{Light curing resins}

Twelve experimental, low-viscosity light curing resins showing different Penetration Coefficients were mixed according to Table 1. A dental adhesive, Excite (Ivoclar Vivadent), was also tested due to its relatively good penetration capability compared to other adhesives observed in a previous study ${ }^{3)}$. Penetration Coefficients of the herein-tested materials have been experimentally determined in a previous study ${ }^{13)}$.

Either Excite or one of the experimental resins was applied on the etched lesions $(n=12)$ using a microbrush. After 10 seconds (B), 22 seconds (C), and 40 seconds (D) respectively, excess resin was wiped away using a cotton roll and the resins were light cured for 30 seconds $\left(530 \mathrm{~mW} / \mathrm{cm}^{2}\right.$; Astralis 5, Ivoclar Vivadent). Subsequently, specimens were cut perpendicularly to the surface (Band Saw Exakt $300 \mathrm{cl}$ ) and cut surfaces were polished plane-parallel (Exakt Mikroschleifsystem, Exakt Apparatebau). A total of 10 lesions broke during the preparation process and were thus excluded.

Confocal laser scanning microscopy

To visualize the pore structures of remaining lesions, 
Table 1 Compositions (weight percent) and Penetration Coefficients ( $\mathrm{PC}$ in $\mathrm{cm} / \mathrm{s}$ ) of tested materials

\begin{tabular}{|c|c|c|c|c|c|c|}
\hline Material & BisGMA* & TEGDMA* & $\mathrm{CQ}^{*}$ & $\mathrm{DABE}^{*}$ & Ethanol* & $\mathrm{PC}^{* * *}$ \\
\hline Excite $^{* *}$ & \multicolumn{4}{|c|}{ not specified } & 25 & 31.3 \\
\hline 1 & 74.25 & 24.75 & 0.5 & 0.5 & - & 0.5 \\
\hline 2 & 66.75 & 22.25 & 0.5 & 0.5 & 10 & 6.0 \\
\hline 3 & 59.25 & 19.75 & 0.5 & 0.5 & 20 & 24.4 \\
\hline 4 & 49.50 & 49.50 & 0.5 & 0.5 & - & 8.8 \\
\hline 5 & 44.50 & 44.50 & 0.5 & 0.5 & 10 & 49.4 \\
\hline 6 & 39.50 & 39.50 & 0.5 & 0.5 & 20 & 91.1 \\
\hline 7 & 24.75 & 74.25 & 0.5 & 0.5 & - & 63.3 \\
\hline 8 & 22.25 & 66.75 & 0.5 & 0.5 & 10 & 129.3 \\
\hline 9 & 19.75 & 59.25 & 0.5 & 0.5 & 20 & 185.4 \\
\hline 10 & - & 99.00 & 0.5 & 0.5 & - & 204.1 \\
\hline 11 & - & 89.00 & 0.5 & 0.5 & 10 & 273.1 \\
\hline 12 & - & 79.00 & 0.5 & 0.5 & 20 & 390.7 \\
\hline \multicolumn{7}{|c|}{$\begin{array}{l}\text { *: Components for experimental infiltrants were purchased from Sigma Aldrich, Steinheim, Germany. } \\
\text { BISGMA: bisphenol A glycerolate dimethacrylate; TEGDMA: triethylene glycol dimethacrylate; CQ: } \\
\text { camphorquinone; DABE: ethyl 4-(dimethylamino)benzoate. } \\
\text { **: Excite (light curing bonding agent; Ivoclar Vivadent, Schaan, Liechtenstein) contains phosphonic acid acrylate, } \\
\text { hydroxyethyl methacrylate, bisphenol A glycerolate dimethacrylate, glycerol dimethacrylate, urethane } \\
\text { methacrylate, ethanol, silicon dioxide, initiators, stabilizers (manufacturer's information). } \\
\text { ***: Penetration Coefficients were determined in a previous study }{ }^{13} \text {. }\end{array}$} \\
\hline
\end{tabular}

specimens were immersed in $50 \%$ ethanol solution containing $100 \mu \mathrm{M}$ sodium fluorescein (SF; Sigma Aldrich) for 10 minutes. Subsequently, specimens were thoroughly washed in deionized water for three minutes and observed using a confocal laser scanning microscope (CLSM; TCS-NT, Leica, Heidelberg, Germany) in combined fluorescence and transmission mode. Excitation light had a maximum wavelength of $488 \mathrm{~nm}$, and fluorescence was detected using a $525 / 50 \mathrm{~nm}$ bandpass filter. Transmitted light was detected without filters. Images were recorded with a lateral resolution of $1024 \times 1024$ pixels and $1000 \times 1000 \mu \mathrm{m}$, and analyzed using Leica Confocal Software (LCS Lite version 2.61, Leica).

At four defined points per lesion indicated by a $100-\mu \mathrm{m}$ grid, both lesion depth and penetration depth were measured and their mean values calculated. Lesion depth was measured for each lesion individually. In cases of complete infiltration, the original lesion depth could not be determined. As such, lesion depth of the corresponding untreated control would be used to calculate percentage penetration.

\section{Statistical analysis}

Statistical analysis was performed using SPSS software (SPSS for Windows version 11.5.1, SPSS Inc., Chicago, IL, USA). Data were checked for normal distribution with Kolmogorov - Smirnov test. Differences in penetration depth between various penetration times were analyzed using Wilcoxon test.
Regarding lesion depth and penetration depth, various materials were compared using Mann Whitney test. Correlation analysis was performed to check validity of the Washburn equation (Pearson's correlation coefficient). Level of significance was set at $5 \%$.

\section{RESULTS}

In the confocal microscopic images, porous structures (i.e., enamel lesions) appeared green due to imbibition of SF solution. Occasionally, the fluorescent dye accumulated at the progressing lesion front or beneath the resin layer, leading to slightly increased fluorescence in these regions. In contrast, solid structures like sound enamel or infiltrated lesion parts showed no fluorescence. However, resininfiltrated zones within the lesion bodies were not homogeneous in all specimens. In all groups, cleftlike porosities showing green fluorescence could be frequently observed within the resin layer (Fig. 1).

Mean lesion depth (standard deviation) in the untreated controls was 294 (58) $\mu \mathrm{m}(\mathrm{n}=146)$. As shown in Table 2, no significant differences in lesion depth could be found among the test groups ( $p>0.05$, Mann - Whitney test).

Figure 2 shows the penetration depths for the test resins at different penetration times. After 10second application, materials 10 [280 (58) $\mu \mathrm{m}], 11$ $[273(47) \mu \mathrm{m}]$, and $12\left[\begin{array}{lll}305 & \text { (97) } \mu \mathrm{m}\end{array}\right]$ showed the 
No. 10
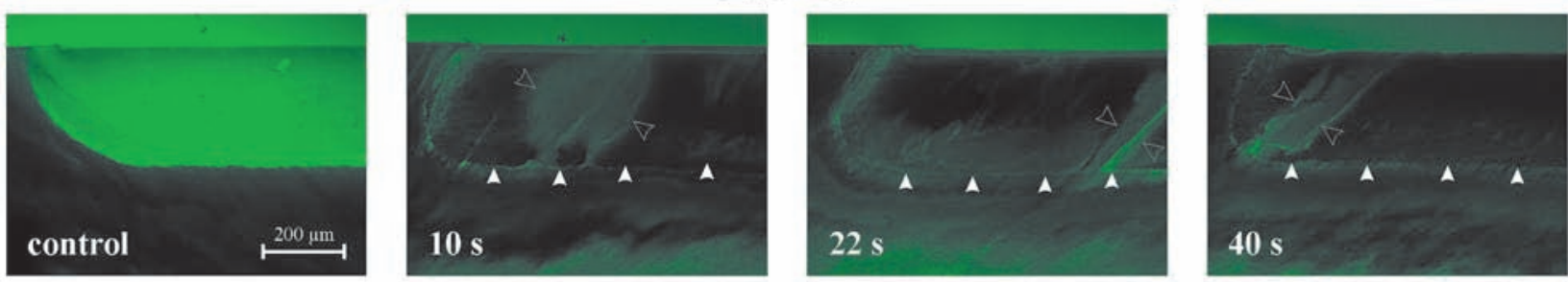

\section{Excite}
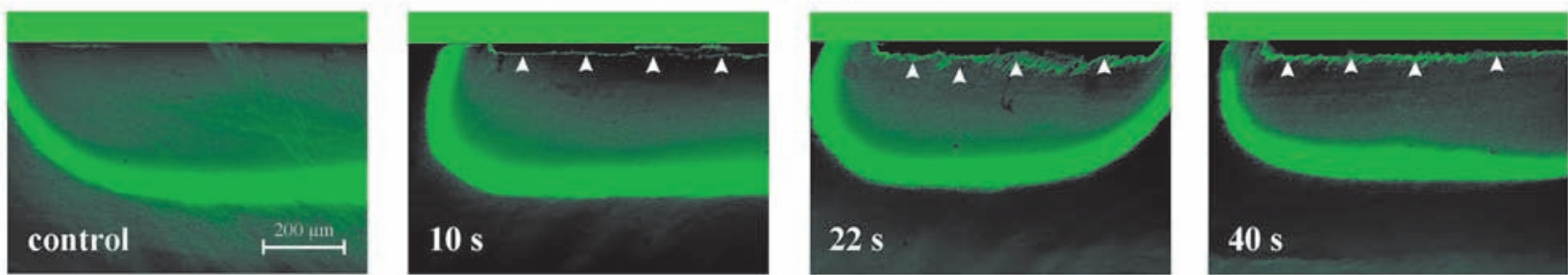

Fig. 1 Representative CLSM images of a specimen treated with material No. 10, showing complete infiltration at all three application times (penetration front is indicated by filled arrowheads). However, the resin layer was interspersed with inhomogeneities (open arrowheads). Penetration of Excite was only superficial (for better visualization, the area above the specimen surface was masked green). Note the accumulation of fluorescent dye at the progressing lesion front.

Table 2 Lesion depths of untreated control lesions $(\mu \mathrm{m})$ and percentage penetration depths in relation to lesion depths (\%) for various application times

\begin{tabular}{|c|c|c|c|c|c|c|c|c|c|c|c|}
\hline \multicolumn{3}{|c|}{ Lesion depth } & \multicolumn{9}{|c|}{ Percentage penetration depth } \\
\hline \multirow[t]{2}{*}{ Material } & \multicolumn{2}{|c|}{ Untreated control } & \multicolumn{3}{|c|}{$10 \mathrm{~s}$} & \multicolumn{3}{|c|}{$22 \mathrm{~s}$} & \multicolumn{3}{|c|}{$40 \mathrm{~s}$} \\
\hline & Mean & $\mathrm{SD}$ & Mean & $\mathrm{SD}$ & sig. & Mean & $\mathrm{SD}$ & sig. & Mean & $\mathrm{SD}$ & sig. \\
\hline Excite $^{\circledR}$ & 291 & 70 & 13 & 7 & $\mathrm{C}$ & 23 & 12 & $\mathrm{EF}$ & 40 & 29 & $\bar{J}$ \\
\hline 1 & 282 & 63 & 6 & 3 & $\mathrm{C}$ & 8 & 3 & $\mathrm{~F}$ & 11 & 4 & $\mathrm{~J}$ \\
\hline 2 & 285 & 39 & 9 & 5 & $\mathrm{C}$ & 16 & 13 & $\mathrm{~F}$ & 21 & 17 & $\mathrm{~J}$ \\
\hline 3 & 266 & 40 & 9 & 2 & $\mathrm{C}$ & 16 & 7 & $\mathrm{~F}$ & 19 & 10 & $\mathrm{~J}$ \\
\hline 4 & 303 & 72 & 10 & 3 & $\mathrm{C}$ & 14 & 8 & $\mathrm{~F}$ & 26 & 19 & $\mathrm{~J}$ \\
\hline 5 & 317 & 33 & 16 & 5 & $\mathrm{C}$ & 45 & 15 & $\mathrm{E}$ & 78 & 21 & I \\
\hline 6 & 291 & 61 & 18 & 11 & $\mathrm{C}$ & 44 & 25 & $\mathrm{E}$ & 81 & 17 & $\mathrm{HI}$ \\
\hline 7 & 253 & 52 & 10 & 4 & $\mathrm{C}$ & 21 & 11 & $\mathrm{EF}$ & 39 & 19 & J \\
\hline 8 & 314 & 28 & 62 & 31 & B & 101 & 24 & $\mathrm{D}$ & 97 & 27 & GHI \\
\hline 9 & 303 & 84 & 81 & 34 & $\mathrm{AB}$ & 106 & 25 & $\mathrm{D}$ & 111 & 21 & $\mathrm{GH}$ \\
\hline 10 & 307 & 67 & 100 & 10 & $\mathrm{~A}$ & 97 & 12 & $\mathrm{D}$ & 107 & 27 & GHI \\
\hline 11 & 288 & 64 & 105 & 11 & A & 118 & 26 & $\mathrm{D}$ & 116 & 26 & $\mathrm{G}$ \\
\hline 12 & 320 & 36 & 104 & 34 & $\mathrm{~A}$ & 112 & 22 & $\mathrm{D}$ & 107 & 28 & GHI \\
\hline
\end{tabular}

Significant differences (sig.) between materials are indicated with different letters ( $\mathrm{p}<0.05$; Mann - Whitney test).

deepest penetration. Concomitantly, these infiltrants completely infiltrated the lesion body (Table 2). After 22-second application, materials 8 [304 (56) $\mu \mathrm{m}]$ and $9[304(65) \mu \mathrm{m}]$ showed complete infiltration of the lesion body as well. None of the other materials was able to penetrate the lesion body completely after 40 seconds. The commercially available adhesive, Excite, showed only 40 (30)\% penetration of the lesion depth after 40-second application. Nonetheless, extension of penetration time from 10 seconds to 22 seconds resulted in significantly increased penetration depths for materials Excite, 2, 3, and 5-9 ( $<<0.05$, 


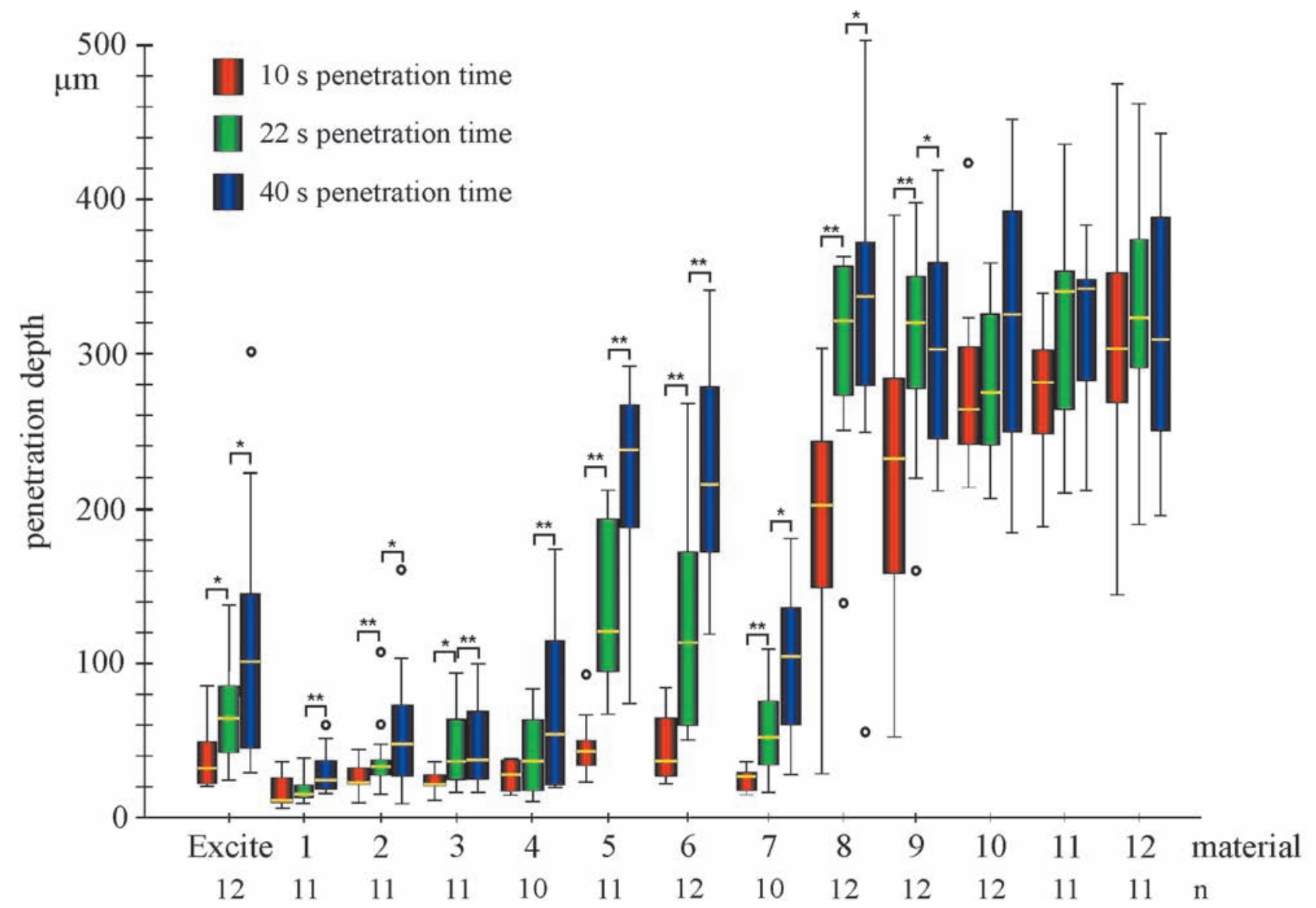

Fig. 2 Penetration depths $(\mu \mathrm{m})$ of the materials after 10-second, 22-second, and 40-second application times (box-andwhisker plots giving quartiles and medians). Significant differences between application times are indicated with asterisks (*: $\mathrm{p}<0.05,{ }^{* *}: \mathrm{p}<0.01$, Wilcoxon test).

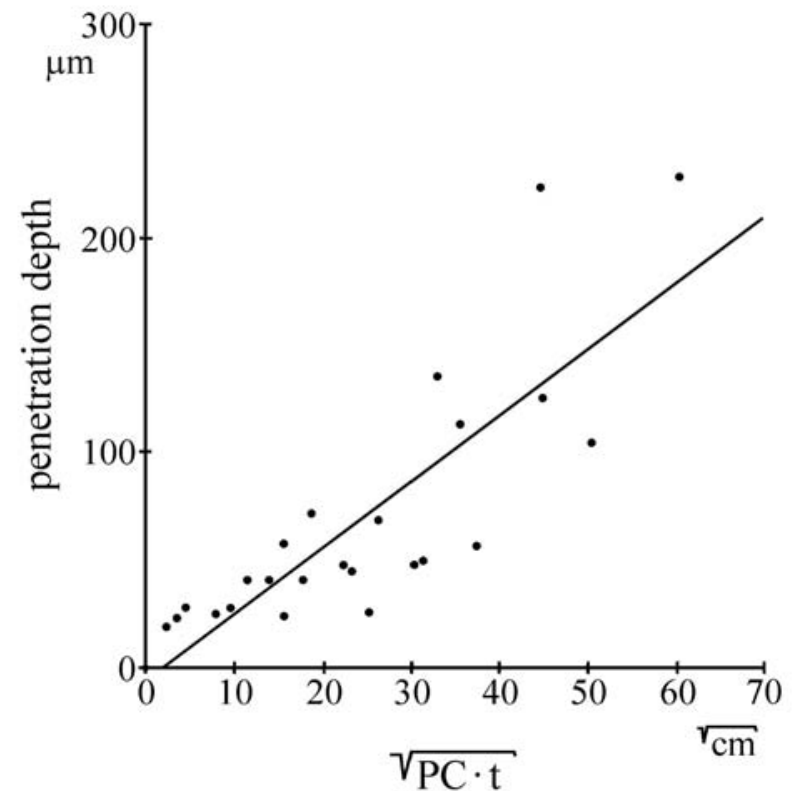

Fig. 3 Scatter plot of penetration depth versus the square root of the product of $\mathrm{PC}$ and application time showing a good correlation $(r=0.820$, Pearson's correlation coefficient).
Wilcoxon test). Further extension of the penetration time to 40 seconds resulted in significantly increased penetration depths for all materials, except for materials $10-12(p<0.05$, Wilcoxon test $)$.

Materials 8-12 were excluded from correlation analysis because of complete infiltration of the lesion body. For Excite and materials 1 - 7, a good linear correlation $(r=0.820, \quad p<0.01$, Pearson's correlation coefficient) between the penetration depth of the corresponding resins and the square root of the product of penetration coefficient and penetration time $(\sqrt{P C \cdot t})$ could be observed (Fig. 3).

\section{DISCUSSION}

Infiltration of enamel caries is a promising therapeutic approach that might bridge the gap between preventive and operative dentistry. However, to date, no materials specially designed for this new purpose are commercially available. Therefore, it was the aim of the present work to explore and identify the material characteristics which allow rapid infiltration of enamel caries. The knowledge of these material characteristics might be 
important for the development of a new group of dental resins, coined as the "infiltrants".

Although an infiltration of the outer layers of the lesion body might inherently delay caries progression, a recent study showed a negative correlation between depth of penetration and progression of caries lesions in a demineralizing environment (unpublished results). Therefore, the aim of any resin infiltration should be the occlusion of preferably as many as possible the pores of caries lesions.

In the present investigation, artificially induced caries lesions were infiltrated. In contrast to natural incipient caries lesions, which are hard to obtain in acceptable numbers, artificial caries lesions can be produced in comparably high numbers. Moreover, due to their relatively uniform structure and lesion depth, the latter seem to be more suitable for the comparison of several materials as compared to natural lesions. However, it must be put into perspective that resin penetration into natural lesions might differ from that observed in artificial caries ${ }^{19}$.

Albeit the relative uniformity of artificial lesions, relatively large variations in lesion depth could be observed in the present investigation. These variations have been reported previously and might be caused by structural differences between various lesions, in terms of lesion depth, pore volume and diameter, as well as surface porosity after acid etching ${ }^{3)}$.

In a previous study, Excite was the fastest penetrating resin of five commercially available adhesives: it infiltrated the lesions (mean $94 \mu \mathrm{m}$ ) completely after 30 seconds ${ }^{3}$. Although showing comparable penetration speed in the present study, Excite was not able to completely infiltrate the lesion body, since current lesions were more than three times deeper (mean $294 \mu \mathrm{m}$ ) compared to the previous ones. For experimental resins 8-12, significantly deeper penetration depths compared to Excite were achieved. Therefore, materials that showed $\mathrm{PC}>100 \mathrm{~cm} / \mathrm{s}$ might be more suitable for application as 'infiltrants' for the treatment of caries lesions compared to fissure sealants and adhesives. It should be noted that materials 8-12 reached their maximum penetration depth (limited by lesion depth) after only 10-second or 22-second application. Thus, the penetration speed of these materials was probably underestimated at longer application times.

In the present study, experimental infiltrants containing high amounts of TEGDMA and ethanol showed the fastest penetration, whereas a high BISGMA content affected penetration capability negatively. Increasing TEGDMA and ethanol contents in TEGDMA/BISGMA/ethanol mixtures have been shown to decrease viscosity and contact angle to enamel, thus leading to increased Penetration
Coefficients ${ }^{13)}$. Improvement of these physical properties might have thus contributed to enhanced capillary action in the enamel lesions of these materials.

At this juncture, it must be pointed that increasing the amount of TEGDMA in BISGMA/ TEGDMA comonomers has been shown to lead to higher polymerization shrinkage ${ }^{20)}$ and brittleness ${ }^{21}$. Moreover, enzymatic hydrolysis stability seems to decrease with increasing TEGDMA content ${ }^{22}$. In the present study, all materials showed zones of imperfect infiltration which might reduce the imperviousness of the infiltrated resin. These inhomogeneities have been reported previously ${ }^{3)}$ and might be caused by oxygen inhibition, polymerization shrinkage, or incomplete evaporation of the solvent. Two-layer application has been shown to reduce such porosities ${ }^{5,7}$.

Results of the present investigation revealed a good correlation between penetration depth and the square root of the product of $\mathrm{PC}$ and penetration time for Excite and experimental materials 1-7. Thus, the working hypothesis could be corroborated for these materials. Results of materials 8-12 were excluded from correlation analysis, since in most specimens these resins completely infiltrated the lesion bodies at all penetration times. Indeed, restriction of penetration by limited lesion depth led to underestimation of penetration speed, and consequently to a distorted correlation analysis.

According to the Washburn equation, application time is as important for penetration depth as the PC. The quadratic relation between penetration time and penetration depth shows that for infiltration of deeper lesions ( $>1 \mathrm{~mm})$, much longer penetration times as used in the present study might be needed. Moreover, penetration speed depends on capillary radius and pore volume of lesions. Therefore, zones of higher mineral content (e.g., the surface layer of natural lesions) might impede penetration.

Furthermore, it has to be taken into consideration that the Washburn equation is only a simplified model of the real situation. For example, the pores of an enamel lesion are not as open as assumed. Thus, entrapped air in the depth of the lesions could hamper resin penetration ${ }^{23)}$. However, this effect (possibly resulting in deceleration of penetration and incomplete infiltration) could not be observed in the present study. Moreover, the pores of natural lesions are most likely to be contaminated or even occluded by organic materials like salivary proteins and food debris. Organic impurities significantly reduce the surface free energy of enamel ${ }^{24}$, and therefore might impede resin penetration. Finally, it is unlikely that the pores within enamel lesions are of uniform diameter at different lesion depths. Thus, penetration speed might depend on lesion 
depth. Nevertheless, the results of the present study showed that the Washburn equation seemed to be applicable to predict resin infiltration of enamel caries lesions.

\section{CONCLUSIONS}

It could be concluded that the ability of a light curing resin to rapidly penetrate enamel lesions could be predicted via its Penetration Coefficient. Therefore, PC determination seemed to be an appropriate means to estimate the penetrativity of potential infiltrants. For complete resin infiltration of artificial enamel caries lesions within a short period, infiltrants should preferably show a high PC exceeding $100 \mathrm{~cm} / \mathrm{s}$.

\section{ACKNOWLEDGEMENTS}

The authors are indebted to Mrs. Jackeline Schaupp and Mr. Michael Stiebritz for their most valuable contribution to the laboratory work of this study, and to Prof. Dr. Harald Stein, Institute for Pathology, CBF, for providing the CLSM. This study was kindly supported by the Deutsche Forschungsgemeinschaft (DFG; PA 1508/1-1).

\section{REFERENCES}

1) Pitts NB, Fejerskov O, von der Fehr FR. Caries epidemiology, with special emphasis on diagnostic standards. In: Dental caries - The disease and its clinical management, Blackwell Munksgaard, Oxford, 2003, pp.141-163.

2) Kidd EAM, van Amerongen JP. The role of operative treatment. In: Dental caries - The disease and its clinical management, Blackwell Munksgaard, Oxford, 2003, pp.245-250.

3) Meyer-Lueckel H, Paris S, Mueller J, Colfen H, Kielbassa AM. Influence of the application time on the penetration of different dental adhesives and a fissure sealant into artificial subsurface lesions in bovine enamel. Dent Mater 2006; 22: 22-28.

4) Paris S, Meyer-Lueckel H, Mueller J, Hummel M, Kielbassa AM. Progression of sealed initial bovine enamel lesions under demineralizing conditions in vitro. Caries Res 2006; 40: 124-129.

5) Meyer-Lueckel H, Mueller J, Paris S, Hummel M, Kielbassa AM. The penetration of various adhesives into early enamel lesions in vitro. Schweiz Monatsschr Zahnmed 2005; 115: 316-323.

6) Gray GB, Shellis P. Infiltration of resin into white spot caries-like lesions of enamel: An in vitro study. Eur J Prosthodont Restor Dent 2002; 10: 27-32.

7) Robinson C, Brookes SJ, Kirkham J, Wood SR, Shore RC. In vitro studies of the penetration of adhesive resins into artificial caries-like lesions. Caries Res 2001; 35: 136-141.

8) Schmidlin PR, Zehnder $\mathrm{M}$, Pasqualetti $\mathrm{T}$, Imfeld $\mathrm{T}$, Besek MJ. Penetration of a bonding agent into deand remineralized enamel in vitro. $J$ Adhes Dent 2004; 6: 111-115.

9) Mueller J, Meyer-Lueckel H, Paris S, Hopfenmuller W, Kielbassa AM. Inhibition of lesion progression by the penetration of resins in vitro: Influence of the application procedure. Oper Dent 2006; 31: 338-345.

10) Paris S, Meyer-Lueckel H, Kiel bassa AM. Resin infiltration of natural caries lesions. J Dent Res 2007; 86: 662-6.

11) Pashley DH, Sano H, Ciucchi B, Yoshiyama M, Carvalho RM. Adhesion testing of dentin bonding agents: a review. Dent Mater 1995; 11: 117-125.

12) Simonsen RJ. Pit and fissure sealant: review of the literature. Pediatr Dent 2002; 24: 393-414.

13) Paris S, Meyer-Lueckel H, Colfen H, Kielbassa AM. Penetration coefficients of commercially available and experimental composites intended to infiltrate enamel carious lesions. Dent Mater 2007; 23: 742-748.

14) Robinson C, Hallsworth AS, Weatherell JA, Kunzel W. Arrest and control of carious lesions: A study based on preliminary experiments with resorcinolformaldehyde resin. J Dent Res 1976; 55: 812-818.

15) Buckton G. Interfacial phenomena in drug delivery and targeting, Harwood Academic Publishers, Chur, Switzerland, 1995, pp.51-53.

16) Fan PL, Seluk LW, O'Brien WJ. Penetrativity of sealants. J Dent Res 1975; 54: 262-264.

17) O'Brien WJ, Fan PL, Apostolides A. Penetrativity of sealants and glazes. The effectiveness of a sealant depends on its ability to penetrate into fissures. Oper Dent 1978; 3: 51-56.

18) Buskes JA, Christoffersen J, Arends J. Lesion formation and lesion remineralization in enamel under constant composition conditions. A new technique with applications. Caries Res 1985; 19: 490-496.

19) Meyer-Lueckel H, Paris S, Kielbassa AM. Surface layer erosion of natural caries lesions with phosphoric and hydrochloric acid gels in preparation for resin infiltration. Caries Res 2007; 41: 223-230.

20) Feilzer AJ, Dauvillier BS. Effect of TEGDMA/ BisGMA ratio on stress development and viscoelastic properties of experimental two-paste composites. J Dent Res 2003; 82: 824-828.

21) Asmussen E. Restorative resins: hardness and strength vs. quantity of remaining double bonds. Scand J Dent Res 1982; 90: 484-489.

22) Munksgaard EC, Freund M. Enzymatic hydrolysis of (di)methacrylates and their polymers. Scand J Dent Res 1990; 98: 261-267.

23) Asmussen E. Penetration of restorative resins into acid etched enamel. II. Dissolution of entrapped air in restorative resin monomers. Acta Odontol Scand 1977; 35: 183-191.

24) Baier RE. Principles of adhesion. Oper Dent 1992; Suppl 5: 1-9. 